\title{
Challenges Found When Patterning Semiconducting Polymers with Electric Fields for Organic Solar Cell Applications
}

\author{
Fernando A. de Castro, ${ }^{1}$ Frank Nüesch, ${ }^{2}$ Christian Walder, ${ }^{2}$ and Roland Hany ${ }^{2}$ \\ ${ }^{1}$ Materials Division, National Physical Laboratory, Hampton Road, Teddington TW11 OLW, UK \\ ${ }^{2}$ Laboratory for Functional Polymers, Swiss Federal Institute for Materials Science and Technology, Empa, Ueberlandstrasse 129, \\ 8600 Dübendorf, Switzerland
}

Correspondence should be addressed to Fernando A. de Castro, fernando.castro@npl.co.uk

Received 26 January 2012; Accepted 11 April 2012

Academic Editor: Mauro Coelho dos Santos

Copyright (C) 2012 Fernando A. de Castro et al. This is an open access article distributed under the Creative Commons Attribution License, which permits unrestricted use, distribution, and reproduction in any medium, provided the original work is properly cited.

A material-independent, contactless structuring method of semiconducting organic materials for the fabrication of interfaceenhanced bilayer solar cells is not available so far. Patterning of thin films using electrohydrodynamic instabilities possesses many desired characteristics and has convincingly been used as a simple method to structure and replicate patterns of nonconducting polymers on submicrometer length scales. However, the applicability of this technique to a wider range of materials has not been demonstrated yet. Here, we report attempts to structure poly( $p$-phenylene vinylene) in a similar way. We found that thin films of poly(2-methoxy-5-(2'-ethylhexyl-oxy)-1,4-phenylene-vinylene) (MEH-PPV) and poly(2-methoxy-5-(3',7'-dimethyloctyloxy)1,4-phenylene-vinylene) (MDMO-PPV) could not be destabilized at all in the limited accessible range of the experimental parameters set by the delicate chemical nature of these materials. We discuss failure origins and present possible loopholes for the patterning of semiconducting polymers using electric fields.

\section{Introduction}

Applications ranging from the medical science to the semiconductor industry require the control of polymer thin film structures on submicron length scales $[1,2]$. A variety of techniques such as lithography or imprinting methods are used for polymer structuring [3]. Some of these methods are still expensive, however, when lower than $100 \mathrm{~nm}$ resolution is required, or may not be applicable for patterning of larger areas. Alternatively, polymers can be structured by self-assembly processes, such as pattern formation via instabilities in thin polymer films or polymer demixing of multicomponent blends [4].

The most efficient organic photovoltaic cells today are based on a bulk heterojunction device geometry consisting of a polymer donor/fullerene acceptor mixture, but the blend nanoscale morphology is difficult to control and depends largely on the processing conditions [5]. Therefore, new approaches that allow the controlled structuring of bicomponent mixtures of semiconducting materials are of general interest [6]. For organic solar cells $[7,8]$, the targeted morphology consists of a bilayer structure $(\sim 200 \mathrm{~nm}$ thick, to absorb the entire incident light) of a donor and acceptor component that interdigitate markedly on a $\sim 20 \mathrm{~nm}$ length scale to enable efficient charge generation within the diffusion length ( $\sim-40 \mathrm{~nm}$ [9]) of the photoexcited electron-hole pair. At the same time, the active components should wet their respective electrode completely, to provide conduction paths for the selective extraction of charges [10] (Figure 1). Concepts for the creation of such nanostructured interface topographical features with high aspect ratios (feature heights: widths) using bottom-up (polymer demixing) or top-down (nanoimprint lithography) have been presented $[11,12]$ (among others), but a general low-cost and largearea compatible pattern method for organic solar cells is not available so far.

With the intention of fabricating such highly folded interpenetrating donor-acceptor bilayers for organic solar cells, we tested here electric fields to destabilize thin films of semiconducting polymers. An electric field applied normal 
to an interface between two dielectric materials polarizes the dielectric, which results in an effective displacement charge density and an interfacial electrostatic pressure. This electrostatic pressure couples to the capillary wave spectrum of the liquid interface, selecting a narrow band of wavelengths that is amplified. Electrohydrodynamic pattering (EHP) has been shown to be an effective means of overcoming interfacial interactions and to produce arrays of nanoscale structures [13]. Using mainly classical, non-conducting polymers so far, electric fields have been applied to induce instability and pattern formation at a liquid/air or at a liquid/liquid interface $[14,15]$. Electric fields have also been used to replicate patterned electrodes with submicron lateral dimensions [16].

\section{Materials and Methods}

Polystyrene (PS, $M_{\mathrm{n}}=150,000$, Aldrich) and poly(2methoxy-5-(3',7'-dimethyloctyloxy)-1,4-phenylene-vinylene) (MDMO-PPV, $M_{\mathrm{n}}=50,000-500,000$, American Dye Source) were used as received, poly(2-methoxy-5-(2'-ethylhexyloxy)-1,4-phenylene-vinylene) (MEH-PPV, $M_{\mathrm{n}}=40,000$ 70,000, Aldrich) was purified before use [11]. Films were spin coated from chlorobenzene $(\mathrm{CB})$ on a first planar silicon electrode $\left(2.5 \times 2.5 \mathrm{~cm}^{2}\right)$ and were dried over night before use. On a second silicon electrode, LiF was evaporated as an $\mathrm{x}$-shaped spacer pattern in high vacuum; afterwards, the two silicon plates were assembled into the capacitor geometry leaving a polymer/air gap. EHP was carried out under vacuum in an oven, with electrical feedthroughs to a Keithley source meter. Experiments were carried out at $T=180^{\circ} \mathrm{C}$, well above the glass transition temperatures and below the decomposition temperatures for both MEH-PPV and MDMO-PPV [17-19]. Rheology measurements (ARES Rheometric Scientific, parallel plate geometry, plate diameter $2.5 \mathrm{~cm}$, plate gap $0.25 \mathrm{~cm}$, shear rates $0.1-100 \mathrm{rad} / \mathrm{s}$, under ambient air) were carried out for PS and MDMO-PPV in the temperature range of $160-240^{\circ} \mathrm{C}$. AFM experiments were carried out on a Nanosurf Mobile S instrument. We used the software WsXM to analyse the AFM images [20].

\section{Results and Discussion}

Figure 2 shows a schematic view of the experimental setup. The polymer film is deposited onto the surface of a bottom electrode and covered with a top electrode, separated at a small distance using an insulating spacer. The whole setup is heated above the glass transition temperature $\left(T_{g}\right)$ of the polymer, and an electric field is applied between the two electrodes. If the electrostatic pressure exceeds the capillary pressure generated by the curvature of the film due to thermal-induced surface fluctuations, the film is destabilized and pillars evolve with a characteristic separation distance $\lambda \propto \gamma^{1 / 2} H^{3 / 2} V^{-1}$ (for planar electrodes), where $\gamma$ is the interfacial tension, $V$ the applied voltage, and $H$ the electrode spacing [13]. Decreasing the temperature below $T_{g}$ finally freezes the morphology.

Since the electric field-induced instability is not material specific, it should be possible to destabilize essentially any

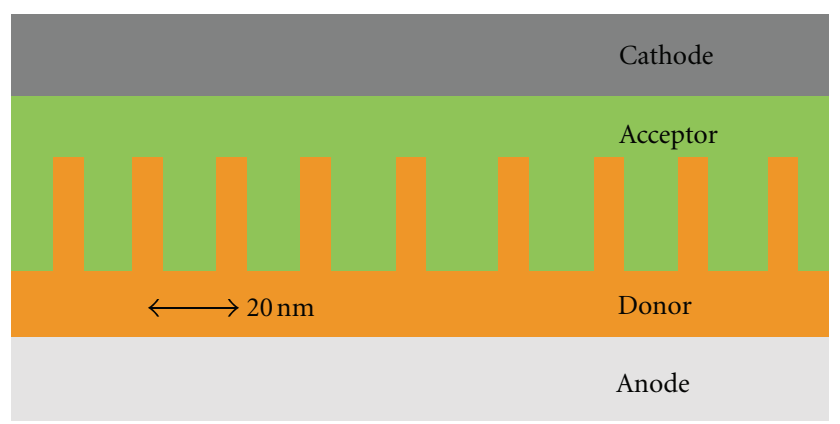

FIGURE 1: Sketch of an idealized (experimentally not yet realized on larger scale) organic heterojunction solar cell. The nature of the organic materials dictates the solar cell design criteria, which implies aspect ratios (height: width) of $>1$ for the nanostructured interface topography.

liquid with reasonably low viscosity, as long as the polymer does not degrade when heated to high temperatures. The experiment involves low-cost equipment and allows to pattern larger areas, provided that the electrodes can be arranged precisely parallel to each other. These are essential prerequisites for organic solar cell applications, where a proof-of-concept device would measure an active area of several square millimetres at least. As an important potential advantage for semiconducting polymers, patterning involves minimal contact between the material and the electrode, whereas lithographic or printing techniques can compromise optical and electrical properties [3].

We compared the effects of electric field-induced destabilization using both insulating (PS) and semiconducting polymers (MEH-PPV and MDMO-PPV). Figure 3(a) displays a destabilized PS film with characteristics closely resembling those reported when using similar experimental parameters. We used planar silicon electrodes separated by a $280 \mathrm{~nm}$ thick LiF spacer. The PS film had a thickness of $100 \mathrm{~nm}$, and a voltage of $25 \mathrm{~V}$ was applied. During a total annealing time ( $T=180^{\circ} \mathrm{C}$ ) of 22 hours, a small dielectric leakage current (9 $\mathrm{mA})$ was flowing through the device [21].

This result indicates that the patterning method is robust and can easily be reproduced. Most often, destabilized features show a correlation over a length of several periods, beyond which domains with different orientations are found [13]. This would be of no concern for photovoltaic applications, where only the local heterojunction interface area should be maximised for charge transfer within the exciton diffusion length [9]. Enlarging the viewing angle for the PS sample, however, revealed that also the wavelengths of destabilized features varied considerably over a distance of $150 \times 150 \mu \mathrm{m}^{2}$ (see Figure $3(\mathrm{~b})$ ). This is probably a signature of small lateral variations of the electric field, introduced by slight variations in the plate spacing [4]. We conclude that the precise electrode adjustment and control of the air gap and capacitor plate distances for larger areas requires a more sophisticated experimental setup than used here, for example, where a gap monitoring scheme is used to fix the electrodes at a constant distance and parallel to each other [22]. In addition, the strong dependence of the developing 


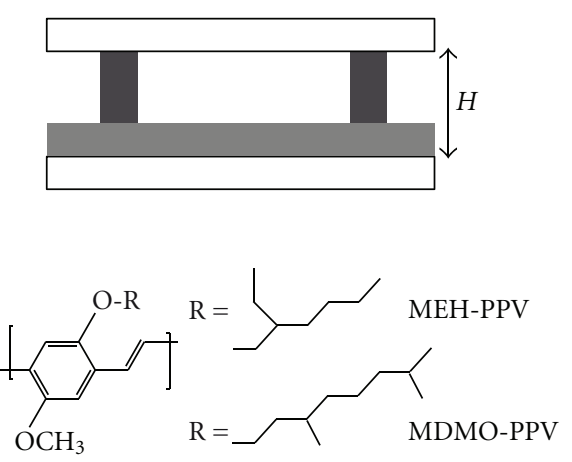

(a)

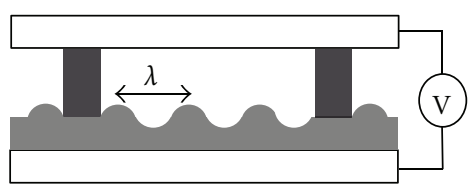

(b)

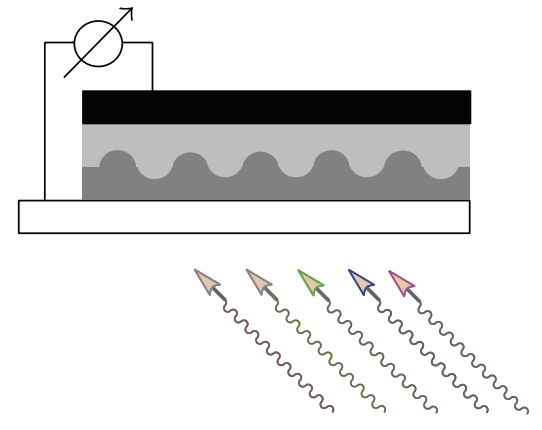

(c)

Figure 2: Molecular structure of the semiconducting polymers MEH-PPV and MDMO-PPV and the experimental EHP setup for the fabrication of organic solar cells. (a) A thin electron-donor polymer film is sandwiched between two electrodes separated by a distance $H$ using insulating spacers. (b) A voltage applied across the electrodes induces instabilities in the film that evolve with a characteristic wavelength $\lambda$. (c) To complete the solar cell, the patterned polymer film is covered by an electron acceptor material and a top electrode. Arrows represent sunlight exciting the solar cell. More elaborated experimental setups could involve the destabilization of donor-acceptor polymer bilayers directly or the guided destabilization via patterned electrodes or prestructured polymer films [11].

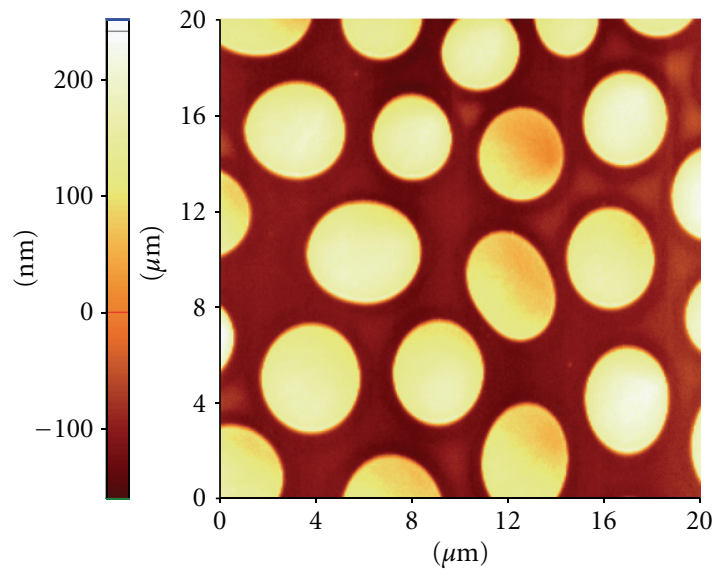

(a)

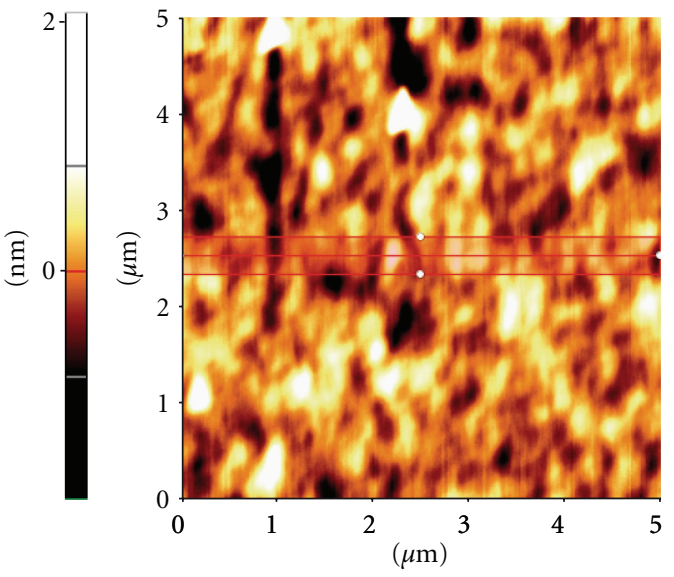

(c)

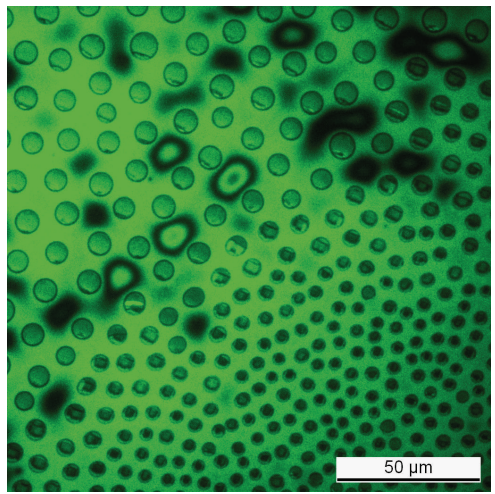

(b)

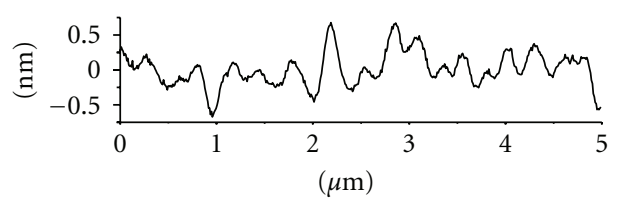

(d)

FIGURE 3: Atomic force microscope (AFM) images of (a) polystyrene (PS) and (c) MEH-PPV films after EHP using the same experimental conditions. Optical microscope image (b) of the patterned PS film. (d) Cross-section height profile of the MEH-PPV film shown in (c). 


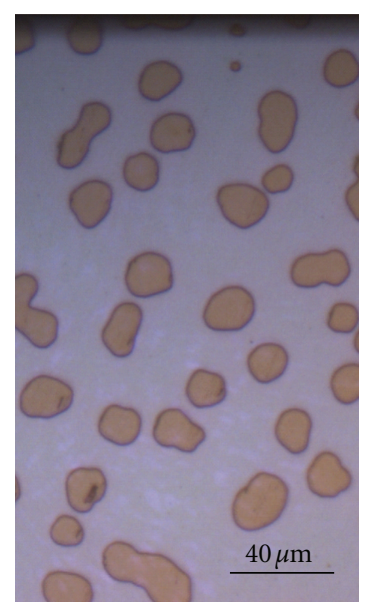

(a)

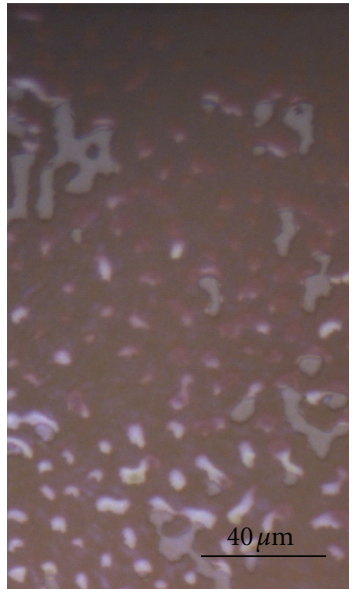

(b)

FIGURE 4: (a) Optical microscopy picture of MEH-PPV/PS bilayer film after EHP processing for 22 hours. PS droplets formed on an underlying MEH-PPV/PS film. (b) Removing PS with the selective solvent cyclohexane reveals that the MEH-PPV film has cracked erratically with no preferred wavelength; black spots indicating polymer degradation could visually be seen in such films.

wavelength on the local distance indicates that the electrodes must be precisely even. This might limit the substrate choice in actual organic solar cell devices, where cost-effective applications in various fields, such as portable electronics or smart textiles, are expected in the near future on flexible devices.

Figure 3(c) shows an AFM image of a treated MEH-PPV film using similar experimental parameters as for PS (100 nm thick film, LiF spacer $280 \mathrm{~nm}, T=180^{\circ} \mathrm{C}, V=20 \mathrm{~V}, t=6 \mathrm{~h}$, leakage current $I=9 \mathrm{~mA}$ ). Apparently, the film did not destabilize and remained essentially flat with roughness below $1 \mathrm{~nm}$ (Figure 3(d)). Attempts with different experimental parameters, such as film thickness $(20,30,80 \mathrm{~nm})$, airgap $(130 \mathrm{~nm})$, or reversed polarity and intensity of the applied electric field, were unsuccessful. The same was observed for the structurally closely related polymer MDMO-PPV. Both PPV polymers were much less stable than PS when exposed to higher voltage and longer destabilization times, what could be visually observed by the development of black spots in the films. This limited the range of parameters that could be used.

The inability to destabilize the semiconducting polymer films was unexpected since at high enough fields, the electrostatic driving force overpowers other interfacial pressures and the materials characteristics should no longer be determining [13]. One reason for the inability to induce instabilities in MEH-PPV and MDMO-PPV might be related to the semiconducting nature of these PPV polymers. While PS behaves to a good approximation as a dielectric and its pattern formation behaviour can be explained by the perfect dielectric models $[13,23]$, charges are more easily injected and rearranged in semiconducting materials. For such materials, the "leaky dielectric model" [24] predicts an additional tangential force at the surface due to the presence of free charges, which will tend to decrease charge concentration. If the normal electrostatic force is capable of overcoming this effect, the presence of free charges is expected to increase the electrostatic stress at the surface, while lowering the electric field in the polymer. This would have little influence on the developing instability wavelength, but the temporal evolution of the instability would be accelerated by several orders of magnitude $[13,21]$. Clearly, these predictions contradict with our experimental results and may suggest that tangential forces are important in these systems.

In a further attempt to destabilize the semiconducting film using EHP, we prepared a PS/MEH-PPV/air gap capacitor device [11]. Hierarchical structure formation using a polymer-polymer-air trilayer system has been convincingly shown using PMMA/PS [14]. Figure 4(a) shows the PS/ MEH-PPV bilayer surface after 22 hour of EHP exposure, and Figure 4(b) displays the remaining MEH-PPV film after selective dissolution of PS. Apparently, PS in the bilayer polymer configuration destabilised in a similar pattern to a film that was coated directly on the silicon electrode (Figure 3(a)) [14]. Again, however, the MEH-PPV film did not follow the expected destabilisation (Figure 4(b)). For shorter process times ( 7 hours), the underlying MEH-PPV film remained flat (roughness below $1 \mathrm{~nm}$, data not shown), while for longer process times the film breaks up erratically, with no preferential feature length scale emerging (not showing features that would suggest an EHP mechanism). In addition, such films changed visually colour and black spots appeared, suggesting that MEH-PPV has degraded.

A further reason that PPV films could not be destabilized might be due to the high viscosity of these stiff, backboneconjugated materials. The developing pattern wavelength itself does not directly depend on the flow behaviour of the polymer melt, but the viscosity $(\eta)$ influences the characteristic time $(\tau)$ for film destabilization strongly [13]. $\tau$ scales linearly with $\eta[25]$ and patterning times ranging from seconds [21] to several days [14] have been reported for polymers with varying $\eta$ values. For PS, the viscosity showed the characteristic thermoplastic flow behaviour, namely, a high 
temperature dependence of $\eta$ for low shear rates, a low temperature dependence of $\eta$ for high shear rates, and a general decrease of $\eta$ with increasing shear rate. On the other hand, MDMO-PPV behaved like a solid, and no meaningful rheological data could be obtained; we also observed that the polymer partly decomposed for temperatures above $200^{\circ} \mathrm{C}$ during the measurement. From this we conclude that MDMO-PPV could not be melted in the accessible temperature range without decomposition, which is probably a signature of the rodlike polymer backbone and the substantial polymer entanglement resulting from the synthesis [26]. Since the $T_{g}$ of MDMO-PPV $\left(\sim 45^{\circ} \mathrm{C}\right)$ [18] is considerably lower than that of MEH-PPV $\left(\sim 66^{\circ} \mathrm{C}\right)[19]$, we argue that the latter would also have a similar solid-like behaviour.

\section{Final Remarks}

Electrohydrodynamic instabilities have been used during the last ten years as a powerful method to pattern polymers with submicron and $\sim 100 \mathrm{~nm}$ features using planar and prestructured electrodes. To this end, mainly insulating polymers of low viscosity at higher temperatures have been used. Here, we tested EHP for the semiconducting polymers MEH-PPV and MDMO-PPV, two of the initial benchmark materials in the emerging field of organic solar cells. While PS could be readily structured using EHP in the most simple and largearea compatible approach, the semiconducting polymer thin films could not be destabilized at all. At the moment, we suspect this to be a combination of the semiconducting nature and/or the viscosity of these materials. The application of even higher temperature or voltage stress to induce destabilization is limited, since semiconducting polymers are labile and their optoelectronic performance degrades quickly with the formation of structural and chemical defects. It might be possible to use alternating electric fields to avoid the tangential movement of charges at the surface and to induce film destabilization. Another approach would be to reduce the polymer viscosity, for instance, by using plasticizers. For example, exposing PS to toluene vapour lowered $T_{g}$ below room temperature and the viscosity was greatly reduced in the swollen film [25].

For possible solar cell applications, the more fundamental issues of creating destabilized features with dimensions well below $\sim 100 \mathrm{~nm}$ and high aspect ratios need to be addressed in greater detail. The aspect ratio of the columnar structures is usually rather small ( $\sim 0.05-0.2[22])$, meaning that the interfacial increase of the donor-acceptor area compared to the planar bilayer configuration in a solar cell would be limited. In principle, by using higher electric fields, both wavelengths can be decreased and the aspect ratio can be increased at the same time. However, due to the electrical breakdown of the capacitor device, this approach is clearly limited. Higher aspect ratios can be created by modulating the electric field distribution using topographically patterned masks. Possibly, this could also be achieved by combining flat electrodes with pre-structured polymer films [11]. In such cases, however, the spatial density of mask (or polymer) pillars is limited. This is because the effective electric field modulation at a fixed distance decreases with increasing density of mask features; a smaller air gap would have to be used in these cases, finally limiting the attainable aspect ratios again [27].

\section{Acknowledgments}

The authors thank Jan Groenewold (University of Utrecht) and Jakob Heier (Empa) for helpful discussions. This paper was supported by Empa and by NPL's Material Research Programme (financed by the UK Department for Business, Innovation and Skills).

\section{References}

[1] L. Jiang, X. Wang, and L. Chi, "Nanoscaled surface patterning of conducting polymers," Small, vol. 7, no. 10, pp. 1309-1321, 2011.

[2] Y. Xu, F. Zhang, and X. Feng, "Patterning of conjugated polymers for organic optoelectronic devices," Small, vol. 7, no. 10, pp. 1338-1360, 2011.

[3] Z. Nie and E. Kumacheva, "Patterning surfaces with functional polymers," Nature Materials, vol. 7, no. 4, pp. 277-290, 2008.

[4] U. Steiner, "Structure formation in polymer films," in Nanoscale Assembly, W. T. S. Huck, Ed., vol. 1, pp. 1-24, Springer, 2005.

[5] Y. Liang, Z. Xu, J. Xia et al., "For the bright future-bulk heterojunction polymer solar cells with power conversion efficiency of 7.4\%," Advanced Materials, vol. 22, no. 20, pp. E135-E138, 2010.

[6] M. Helgesen, R. Søndergaard, and F. C. Krebs, "Advanced materials and processes for polymer solar cell devices," Journal of Materials Chemistry, vol. 20, no. 1, pp. 36-60, 2010.

[7] B. Walker, C. Kim, and T. Q. Nguyen, "Small molecule solution-processed bulk heterojunction solar cells," Chemistry of Materials, vol. 23, no. 3, pp. 470-482, 2011.

[8] P. G. Nicholson and F. A. Castro, "Organic photovoltaics: principles and techniques for nanometre scale characterization," Nanotechnology, vol. 21, no. 49, Article ID 492001, 2010.

[9] P. Peumans, A. Yakimov, and S. R. Forrest, "Small molecular weight organic thin-film photodetectors and solar cells," Journal of Applied Physics, vol. 93, no. 7, pp. 3693-3723, 2003.

[10] P. K. Watkins, A. B. Walker, and G. L. B. Verschoor, "Dynamical monte carlo modelling of organic solar cells: the dependence of internal quantum efficiency on morphology," Nano Letters, vol. 5, no. 9, pp. 1814-1818, 2005.

[11] F. A. Castro, H. Benmansour, C. F. O. Graeff, F. Nüesch, ${ }^{\sim E}$. Tutis, and R. Hany, "Nanostructured organic layers via polymer demixing for interface-enhanced photovoltaic cells," Chemistry of Materials, vol. 18, no. 23, pp. 5504-5509, 2006.

[12] X. He, F. Gao, G. Tu et al., "Formation of nanopatterned polymer blends in photovoltaic devices," Nano Letters, vol. 10, no. 4, pp. 1302-1307, 2010.

[13] N. Wu and W. B. Russel, "Micro- and nano-patterns created via electrohydrodynamic instabilities," Nano Today, vol. 4, no. 2, pp. 180-192, 2009.

[14] M. D. Morariu, N. E. Voicu, E. Schäffer, Z. Lin, T. P. Russell, and U. Steiner, "Hierarchical structure formation and pattern replication induced by an electric field," Nature Materials, vol. 2, no. 1, pp. 48-52, 2003.

[15] P. S. G. Pattader, I. Banerjee, A. Sharma, and D. Bandyopadhyay, "Multiscale pattern generation in viscoelastic polymer 
films by spatiotemporal modulation of electric field and control of rheology," Advanced Functional Materials, vol. 21, no. 2, pp. 324-335, 2011.

[16] E. Schäffer, T. Thurn-Albrecht, T. P. Russell, and U. Steiner, "Electrically induced structure formation and pattern transfer," Nature, vol. 403, no. 6772, pp. 874-877, 2000.

[17] R. F. Cossiello, L. Akcelrud, and T. D. Z. Atvars, "Solvent and molecular weight effects on fluorescence emission of MEHPPV," Journal of the Brazilian Chemical Society, vol. 16, no. 1, pp. 74-86, 2005.

[18] B. Conings, S. Bertho, K. Vandewal et al., "Modeling the temperature induced degradation kinetics of the short circuit current in organic bulk heterojunction solar cells," Applied Physics Letters, vol. 96, no. 16, Article ID 163301, 3 pages, 2010.

[19] J. Y. Kim and C. D. Frisbie, "Correlation of phase behavior and charge transport in conjugated polymer/fullerene blends," Journal of Physical Chemistry C, vol. 112, no. 45, pp. 1772617736, 2008.

[20] I. Horcas, R. Fernández, J. M. Gómez-Rodríguez, J. Colchero, J. Gómez-Herrero, and A. M. Baro, "WSXM: a software for scanning probe microscopy and a tool for nanotechnology," Review of Scientific Instruments, vol. 78, no. 1, Article ID 013705, 8 pages, 2007.

[21] P. Goldberg-Oppenheimer and U. Steiner, "Rapid electrohydrodynamic lithography using low-viscosity polymers," Small, vol. 6, no. 11, pp. 1248-1254, 2010.

[22] M. D. Dickey, A. Raines, E. Collister, R. T. Bonnecaze, S. V. Sreenivasan, and C. G. Willson, "High-aspect ratio polymeric pillar arrays formed via electrohydrodynamic patterning," Journal of Materials Science, vol. 43, no. 1, pp. 117-122, 2008.

[23] E. Schäffer, T. Thurn-Albrecht, T. P. Russell, and U. Steiner, "Electrohydrodynamic instabilities in polymer films," Europhysics Letters, vol. 53, no. 4, pp. 518-524, 2001.

[24] L. F. Pease and W. B. Russel, "Linear stability analysis of thin leaky dielectric films subjected to electric fields," Journal of Non-Newtonian Fluid Mechanics, vol. 102, no. 2, pp. 233-250, 2002.

[25] S. Harkema and U. Steiner, "Hierarchical pattern formation in thin polymer films using an electric field and vapor sorption," Advanced Functional Materials, vol. 15, no. 12, pp. 2016-2020, 2005.

[26] T. Schwalm, J. Wiesecke, S. Immel, and M. Rehahn, “The gilch synthesis of poly(p-phenylene vinylenes): mechanistic knowledge in the service of advanced materials," Macromolecular Rapid Communications, vol. 30, no. 15, pp. 1295-1322, 2009.

[27] J. Heier, J. Groenewold, and U. Steiner, "Pattern formation in thin polymer films by spatially modulated electric fields," Soft Matter, vol. 5, pp. 3997-4005, 2009. 

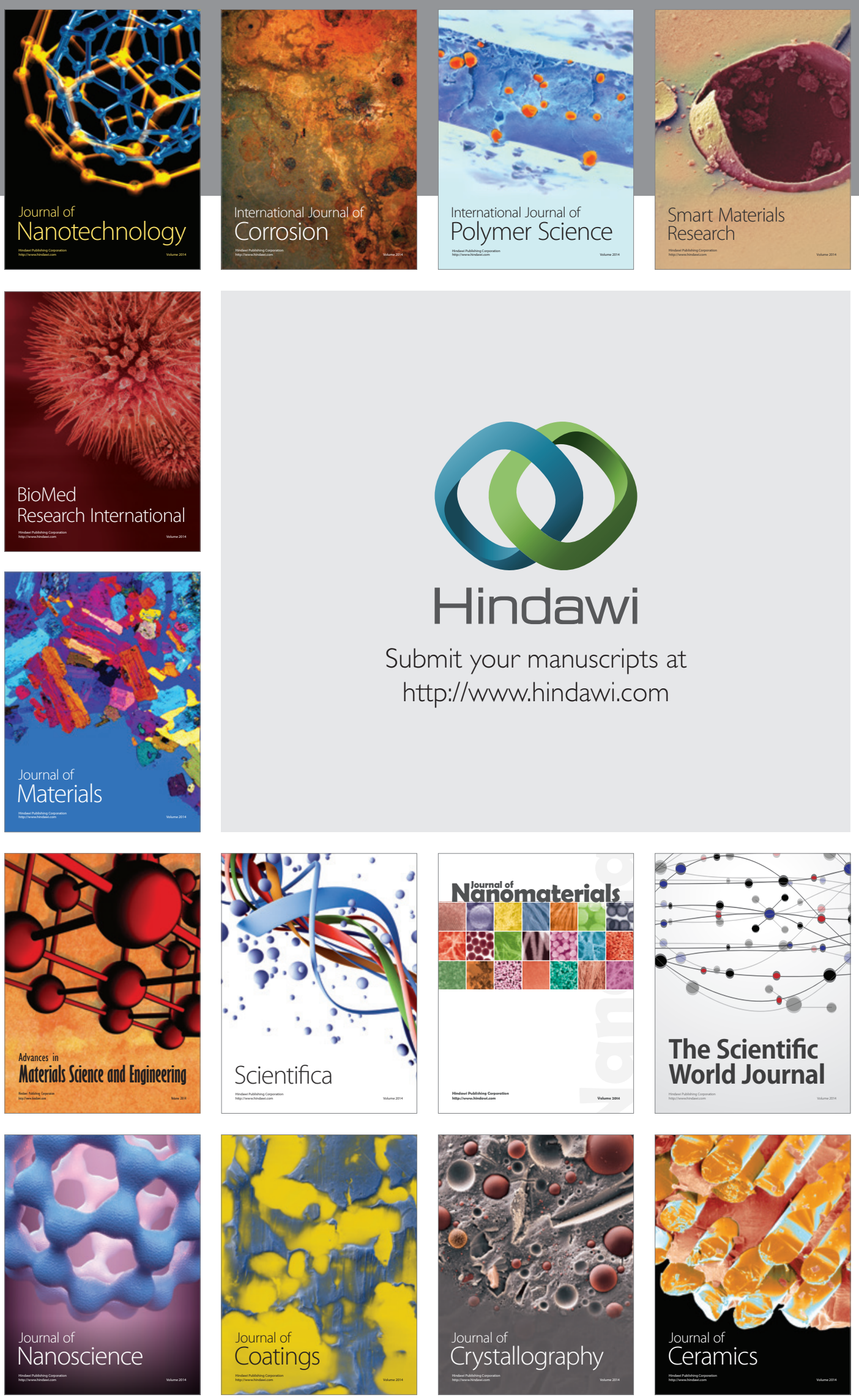

The Scientific World Journal

Submit your manuscripts at

http://www.hindawi.com

\section{World Journal}

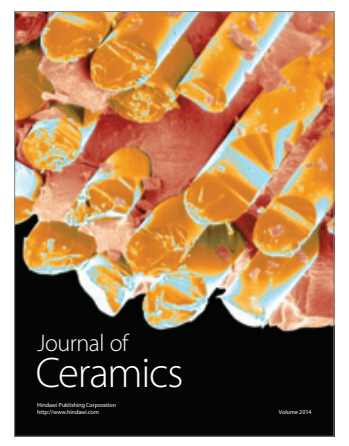

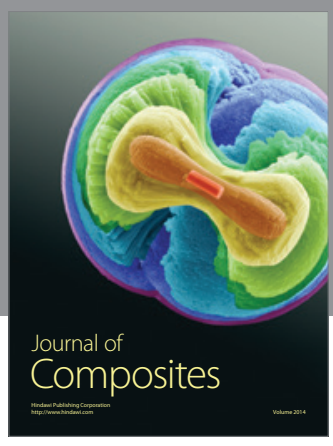
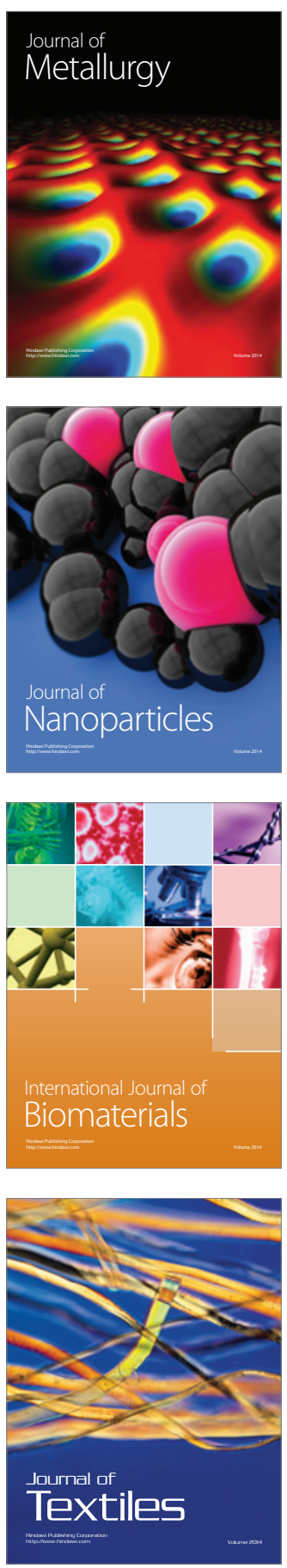